\title{
Reducing Network Energy Consumption using Multidimensional Optimization of LEACH Protocol
}

\author{
Dr.M.Buvana, Dr.K.Muthumayil ${ }^{2}$, Dr.M.Rajinikannan ${ }^{3} \&$ Dr.Jayasankar ${ }^{4}$ \\ ${ }^{1}$ Associate professor, Dept of CSE, PSNACET \\ ${ }^{2}$ Professor, Dept.of IT, PSNACET \\ ${ }^{3}$ Professor, Dept.of Computer Applications, PSNACET \\ ${ }^{4}$ Asst prof, Dept of ECE, University College of Engineering, BIT Campus, Trichy \\ Email: ${ }^{1}$ buvana@psnacet.edu.in, ${ }^{2}$ muthumayil@psnacet.edu.in, ${ }^{3}$ rajinikannan@psnacet.edu.in \\ 4jayasankar27681@gmail.com
}

Article History: Received: 11 January 2021; Accepted: 27 February 2021; Published online: 5 April 2021

\begin{abstract}
The optimized LEACH-focused protocol is proposed to make equal network energy usage and outspread the life cycle. In the first place, the threshold function of the cluster head is adjusted and the technique of division of the network area is configured to change reasonably the size of the clusters, taking account of residual energy and node distance. A sleep mechanism is introduced for intra-cluster transmission to balance the power consumption of nodes. And a new Barycenter Node has been introduced to help the head of the cluster fulfill the transmission task and thus prolong the lifespan. We derived a new formula, considering the angle, energy and distance, in multiple-hop communication between clusters to determine the fitting factor of the next hop. The results of simulations display that the remaining total energy of the optimized LEACH is decreased by $32.6 \%$ and the network life cycle respectively increases by around $54 \%$.
\end{abstract}

Keywords: LEACH Protocol,Sleep Mechanism,Intra Cluster,Barycenter Node,

\section{Introduction}

One of the important remote monitoring tools is the Wireless Sensor Network (WSN). It has significant requests in all walks of life with the advent of the knowledge age. In WSNs, a large number of nodes need to be deployed in the target monitoring area in order to obtain and transmit data. Given the unique character of the monitoring environment, energy conservation by replacing batteries is always difficult for nodes. Consequently, the node life cycle has become the primary norm for designing data acquisition systems of the sensor network[1]. Reasonable routing protocol should be implemented to prolong the network life cycle to solve the problem of restricted node power in WSN. The routing protocol is currently divided primarily into protocol and hierarchical procedure for routing. As planar routing protocols require wide routing tables to maintain and there is limited transmission range between sensor nodes, they are not suitable for large-scale networks, while the hierarchical routing protocol does not only boost these issues, but also decrease energy consumption and increase network life cycles[2]. This paper therefore selects hierarchical protocol routing (LEACH protocol).

LEACH is the chief clustering routing protocol developed by Cui et al., [3] and adopts a hierarchical networking structure. Each node selected the $\mathrm{CH}$ through the election process in order to prolong the existence of the network, and each "circle" in the network will perform the head of the cluster and its reorganization in order to prevent the $\mathrm{CH}$ from failing because of extreme energy consumption.

Following the LEACH protocol, several optimization experiments were undertaken and DEEC [4 and 5] were proposed, as well as other methodologies. In [6], the GA-LEACH Routing Protocol, which combines the micro genetic algorithm with the Leach Protocol, is proposed for improving selection of the cluster head $(\mathrm{CH})$, and for reducing energy consumption in the network, to address the deficiency of the classic clustering LEACH Protocol. In [7], the leach-F algorithm was projected to pick $\mathrm{CH}$ in any round of cluster reconstruction to lessen the energy loss. The author [8] have suggested the non-uniform EEUC clustering algorithm. Distance to the portal node that protects the hot nodes, equalizes energy consumption, are described in clusters of various sizes. A distributing automatic learning algorithm was built in [9]. When each node was selected as a subset of network nodes, at least one active node was protected to attain the network's global objective, and each node was self-protected and thus extended the nodal life cycle. The protocol on cluster head substitution (MT-CHR) in Article [11] was amended. The proposal to be a new cluster head for every node in every round was reasonably consistent with the LEACH protocol assumptions. The proposal proposes a new term for the threshold energy, taking into account the delay of first node death and the avoidance of any data. 
The technology H-LEACH was proposed in literature[10] to resolve the energy problem when the $\mathrm{CH}$ were chosen. For channel heads the threshold conditions were used, the remaining and maximum energy of the nodes were taken into consideration in each round. The article[11] proposed a greedy partial coverage algorithm (GPC), using neighbor nodes to preserve selected node connectivity, using the overlapping of nodes to achieve the necessary coverage rate, improving coverage and connectivity of node to effectively cut network energy consumption. In [12], the new LEACH Protocol was proposed based on affinity propagation, allowing a completely distributed control of the traditional LEACH-based protocols and resolving practical limitations by simplifying the functionality of network networks, and reducing sensor hardware costs [13].

An efficient $\mathrm{CH}$ selection algorithm, PSO-ECHS, based on the optimisation of particulate swarm, and an effective coding and fitness function scheme have been proposed to increase the energy efficiency of the optimization of particulate swarm. In [14] it focused on recent routing protocols that depended on the LEACH protocol to increase the efficiency and life of the WLN. The algorithm of node rank is dependent on the cost of path and the sum of links between cluster heads.

In Article [15] the algorithm pDCD was proposed which was a PCP automatic learning algorithm. It found nodes in the deployed network to track the p percentage coverage, achieving the maximum network coverage and improving the network life cycle significantly. An improved energy-saving protocol (IEE-LEACH) was projected in [16], taking into account the energy of the other nodes and the average network energy. The nodes close to the base station cannot connect to the cluster for the optimum number of cluster heads, which has decreased wireless network energy use significantly.

\section{2. Optimization ofLEACH Protocol}

\subsection{LEACH Procedure}

LEACH is a mechanism is that the $\mathrm{CH}$ is taken over by all nodes in turn. The cluster heads are designed to collect and send information before the Gate Node to the next head of cluster (GN). In general, cluster heads have a much higher energy consumption than ordinary nodes. The rotary mechanism of the CHs thus prevents unnecessary consumption and death of the $\mathrm{CH}$, equalizes the energy consumption between nodes and extends the network life cycle. Those who served as cluster leaders no longer contribute in the elections before any selection of cluster leaders.

Suppose a node randomly chooses a number from 0 to 1 . At the beginning, if the random number generated by a node is less than $T(n)$, it will become the cluster head of the current round. The appearance of $T(n)$ is as follows:

$$
T(n)=\left\{\begin{array}{c}
\frac{P}{1-P \times\left(r \bmod \left(\frac{1}{P}\right)\right)}, \text { if } n<G \\
0 \quad \text { otherwise }
\end{array}\right.
$$

In equation (1), $r$ is the present rounds, $P$ is the percentage of nodes which become $\mathrm{CH}$, and $G$ is identified as the set of nodes, which don not chosenCHs in the $1 / P$ round before the present round.

\subsection{Energy Model Scheme}

Figure 1 and formula (2) correspondingly display the node contact model and power consumption formula of WSN.

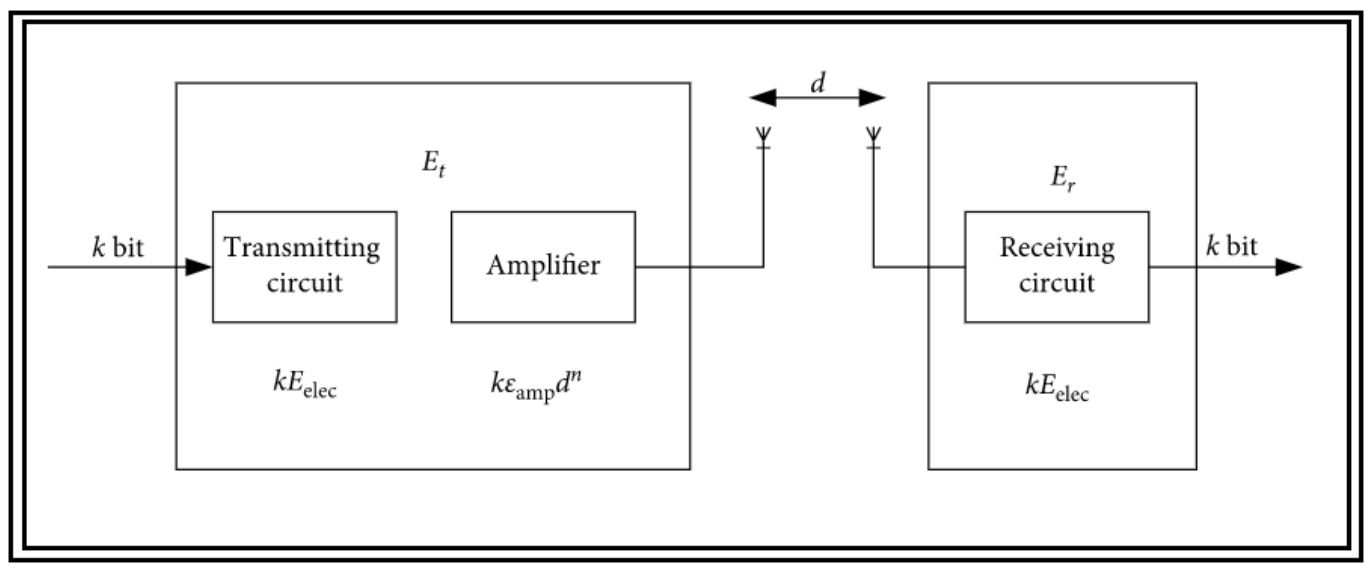

Figure 1: Model diagram of node communication.

$$
\begin{aligned}
& E_{t}=\left\{\begin{array}{c}
K E_{\text {elec }}+x K \varepsilon_{f s} d^{2}, d \leq d_{0} \\
K E_{\text {elec }}+x K \varepsilon_{\text {amp }} d^{4}, d \geq d_{0}
\end{array}\right. \\
& d_{0}=\sqrt{\frac{e_{f s}}{\varepsilon_{a m p}}}
\end{aligned}
$$


In equation (2), $d \leq d_{0}$ is a free space model and $d \geq d_{0}$ is a multipath attenuation model. In universal, intracluster calculations use $d^{2}$ and intercluster transmission. $e_{f s}$ is receiver sensitivity related amplification. $E_{\text {elec }}$ is magnification energy connected to the received noise image. $\varepsilon_{a m p}$ is electricity related to factors such as digital coding.

Agreeing to the above, the received energy consumption $E_{r}$ can be distinct as

$$
E_{r}=k E_{\text {elec }}
$$

The energy use of WSN comprises, primarily, energy transmission consumption, processing, idle energy use and consumption of sleep. Research demonstrates that the key energy loss is receiving and transmitting energy. Furthermore, several redundant nodes appear during data transmission. Their energy efficiency is much higher than that of sleep and the sleep system is used to absorb energy in nodes in the cluster.

\section{3. Experiment and Discussion}

The optimized LEACH protocol is associated to LEACH and LEACH-C in the same simulation environment [17]. In the same number of rounds, they primarily compare the sum of dead nodes, the sum of data packets, and the network energy remaining.

Suppose 200 nodes distributed in the $200 \sim 200$ plane is composed of the network. When ten percent of the initial energy was left of the node, the node died. The optimized LEACH protocol represented I-LEACH in all simulation figures in this article.

The LEACH activity protocol is table 1 and the simulation experimentation parameters are table 2 . Table 2.

Table 1: The running process of LEACH.

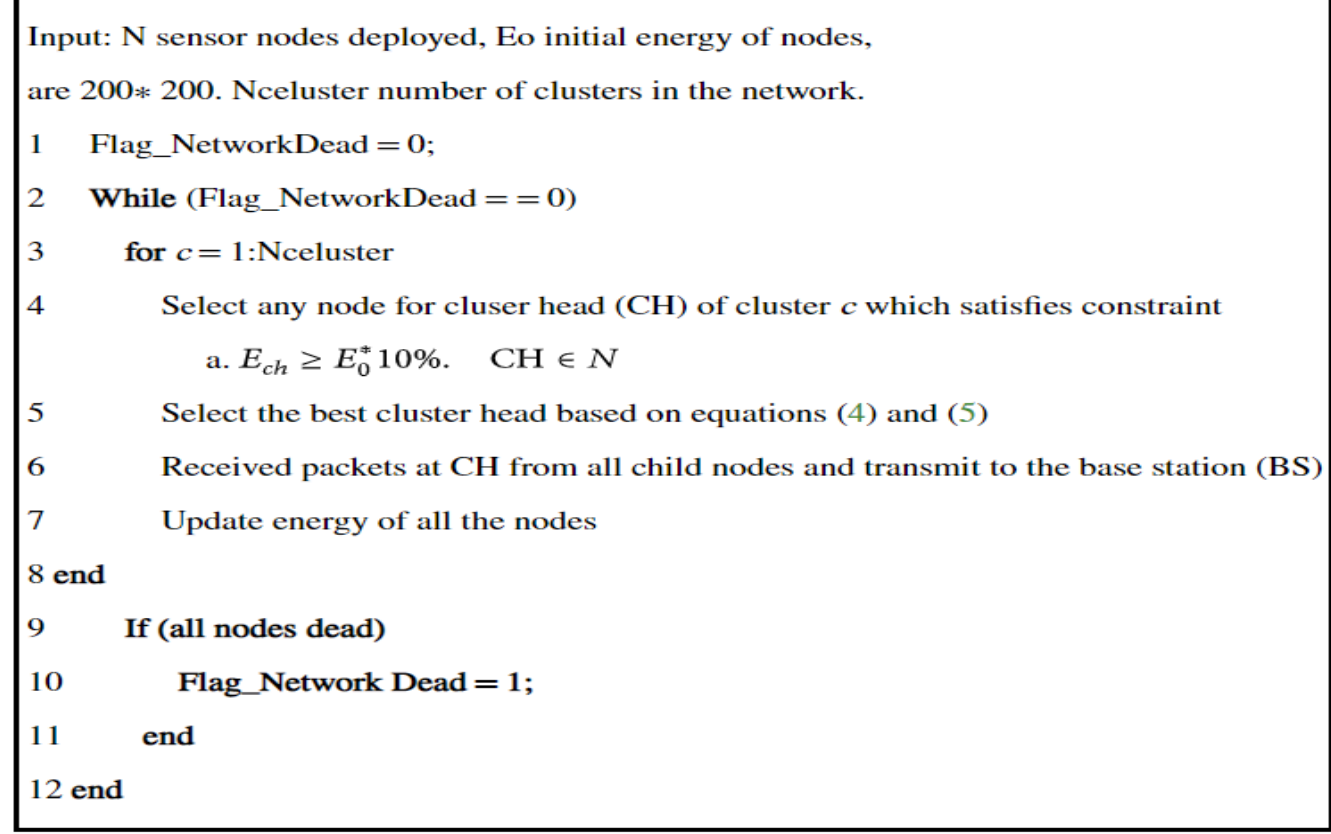

Table 2: parameters for Simulation.

\begin{tabular}{|l|c|}
\hline Parameter & Parameter value \\
\hline Network size & $200 \mathrm{~m} \times 200 \mathrm{~m}$ \\
Number of nodes & 200 \\
$E_{\text {elec }}$ & $50 \mathrm{~nJ} / \mathrm{bit}$ \\
$\varepsilon_{f s}$ & $100 \mathrm{pJ} /\left(\mathrm{bit} \cdot \mathrm{m}^{2}\right)$ \\
$\varepsilon_{\text {amp }}$ & $0.0013 \mathrm{pJ} /\left(\mathrm{bit} \cdot \mathrm{m}^{4}\right)$ \\
Initial energy & $0.5 \mathrm{~J}$ \\
$\mathrm{~d}_{0}$ & $87 \mathrm{~m}$ \\
Packet size & $4000 \mathrm{bits}$ \\
\hline
\end{tabular}




\section{4 Comparison of the simulation results}

As Figure 2 shows, when a protocol is running 1500 rpms, the curve shows the comparison of living nodes. The abscissa shows the sum of rounds, and the ordinate shows the sum of nodes living. Figure 6 shows that in 1500 rounds, the number of LEACH optimized live nodes is essentially much more than LEACH. In comparison to the LEACH-C protocol [17], there is no difference between the number of live nodes before the 700 LEACH optimized protocol rounds. It's clearly more than that of LEACH-C after 700 shots. This is because the lower energy nodes are covered and the energy consumption of the network balanced during the electoral point, by improving the electoral feature. The optimized LEACH protocol is typically considered to secure node lives, so the live number of the node is better than the other two algorithms.

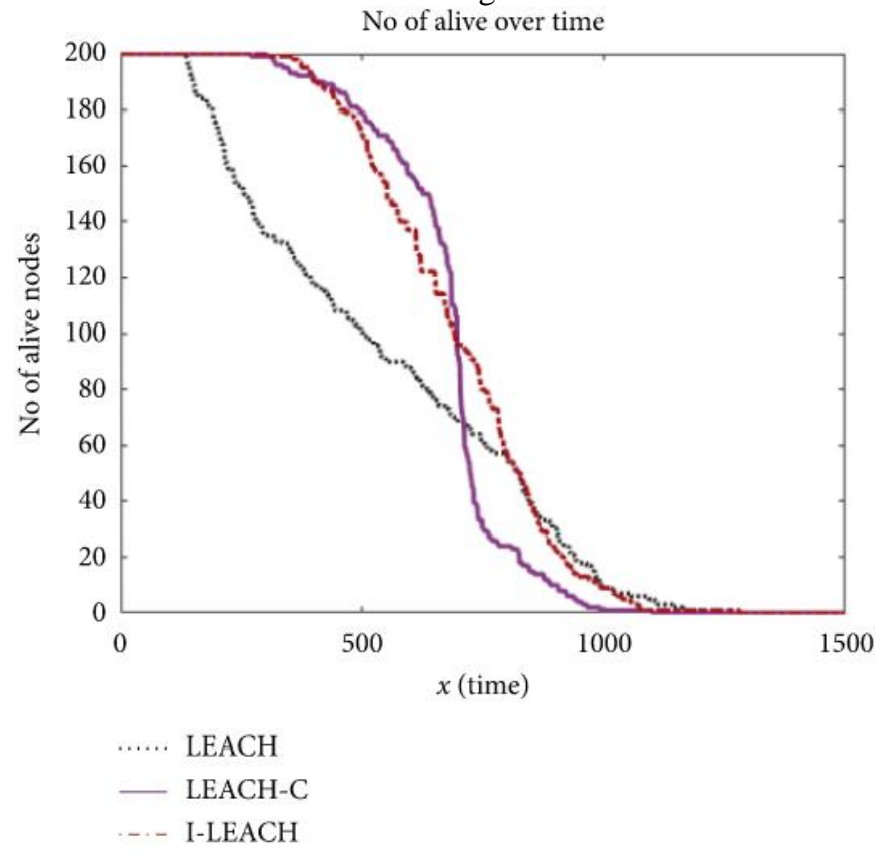

Figure 2: Comparison of the sum of alive nodes under the three protocols.

As exposed in Figure 3, the curve compares the number of dead nodes at 1500 rounds of the protocol. The number of rounds is abscissa, and the number of dead nodes is ordinate. Between LEACH-C[17] and optimized LEACH, the first dead node appears around 400. In terms of the sum of dead nodes, the optimized LEACH and LEACH-C protocol before 700 rounds perform considerably better than LEACH and there is little difference among these two protocols. LEACH nodes die slightly lower than the other two algorithms after seventy hundred rounds. Overall, there is a balanced death rate of optimized LEACH nodes which maximizes node life. The LEACH C optimized protocol is designed to postpone the first dead node by improving choice and introducing new threshold features to allow additional nodes to operate up until the end, to achieve network growth balance and to improve the scalability of the WSN system. 


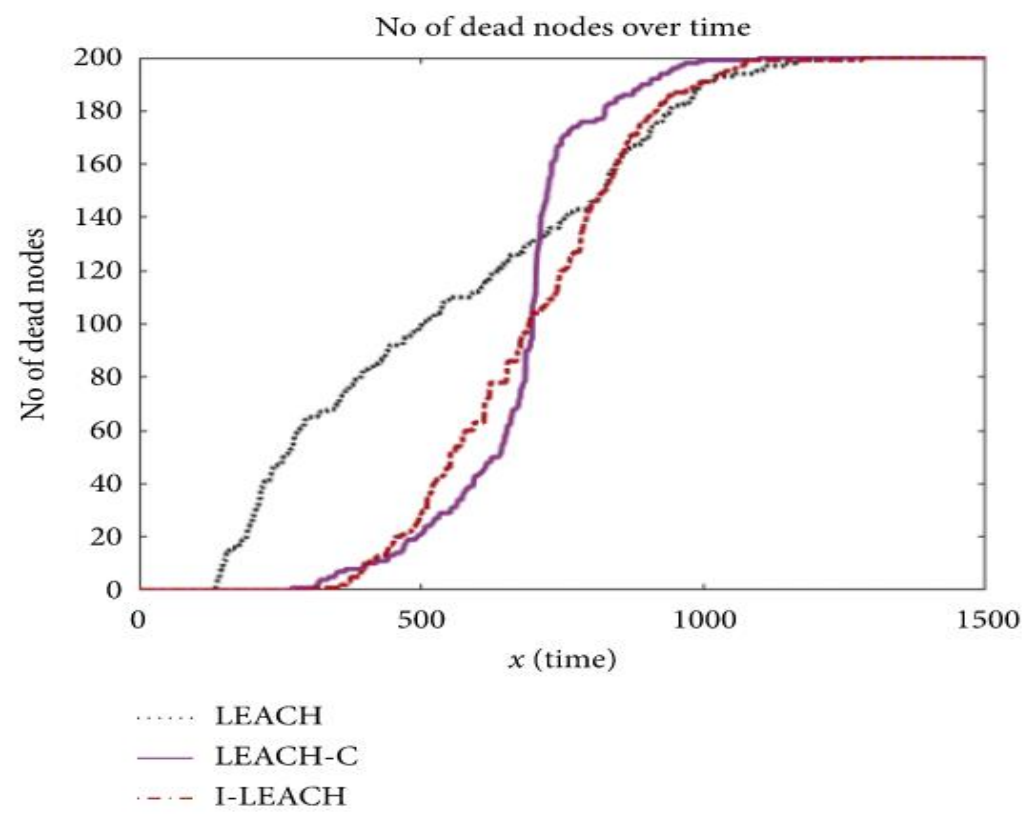

Figure 3: Comparison of the sum of dead nodes under the three protocols.

Energy consumption of nodes is provided mainly when data packets are collected and sent. Long-term vitality for wireless sensor networks can only be achieved by balanced energy consumption between nodes. The load should be distributed uniformly between the nodes. Figure 4 demonstrates the network's residual energy in 1500 cycles in the three protocols. The optimized protocol, as opposed to LEACH, is around 30 per cent more than the total residual energy of original LEACH, and 15 per cent more than LEACH-C[18], whereas the lower consumption and better balance in terms of energy are a linear relationship. Figure 8 shows that the residual node energy distribution is uneven and shows that LEACH protocol has Polar Weathering.

The total remaining energy of the system in J
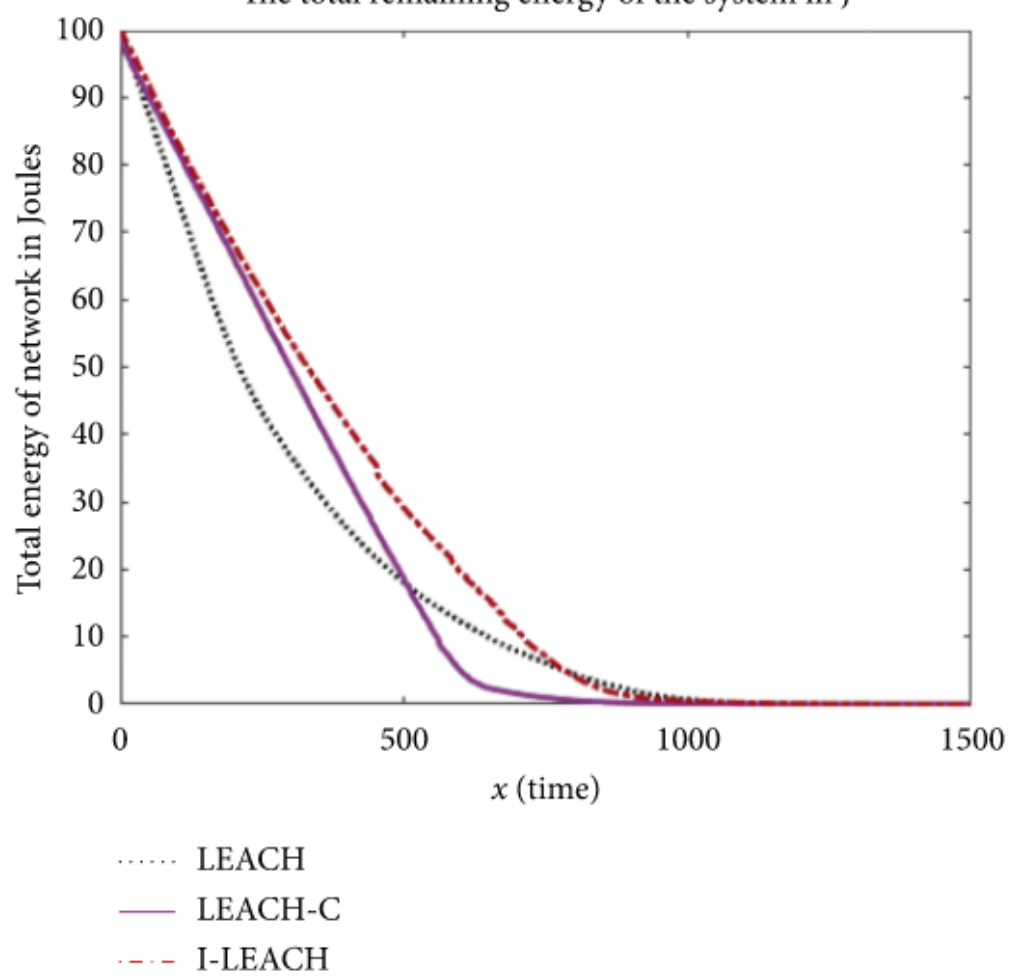

Figure 4: Residual energy of the network under the three protocols.

The changing value of the original threshold function particularly after the death of the first node provides every node with a chance of being classified headers, thus increasing randomness and increasing energy use. Some nodes die prematurely, while several nodes are strong. It optimizes the $\mathrm{CH}$ selection with regard to node residual energy and nodes consume energy uniformly in the network; almost every node of the network is energy efficient. For the same initial energy, the simulation results are compared with [3]; the nodes have a higher 
energy and a better convergence rate at any given time. The optimized LEACH protocol therefore improves the energy balance and increases the network life cycle.

4. 5. Conclusion

This article examines the inconvenience of LEACH's current protocol in a thorough way and then enhances its creation of clusters, its transmission within clusters, cluster contact, auxiliary transmission and other links in the LEACH. Through comparing simulations, the number of nodes that survive, the network life-cycle and residual energy networks are shown to have great advantages. However, it has only more than 300 rounds of time, which is not ideal and restricts the reach of operation considerably, based on the rounds when all nodes die. Future research objectives are to increase network efficiency when all knots die, reduce energy consumption and prolong the network life cycle. We also strive to put an integrated LEACH network and heterogeneous real-time environment.

\section{References}

1. M. Radhika and P. Sivakumar, "Energy optimized micro genetic algorithm based LEACH protocol for WSN,” Wireless Networks, vol. 8, no. 1, 2020.

2. P. Sivakumar and M. Radhika, "Performance analysis of LEACH-GA over LEACH and LEACH-C in WSN," Procedia Computer Science, vol. 125, no. 1, pp. 248-256, 2018.

3. L. Wang, B. J. Xie, Z. Z. Liu et al., "Improved algorithm for non-uniform clustering routing protocol," Computer Scince, vol. 44, no. 2, pp. 152-156, 2017.

4. H. Mostafaei and M. S. Obaidat, "A distributed efficient algorithm for self-protection of wireless sensor networks," in Proceedings of the 2018 IEEE International Conference on Communications (ICC), IEEE, Kansas City, MO, USA, May 2018.

5. L. Deng-Ao, H. Hai-Long, G. Jin-Long et al., "Clustering and Inter-cluster Routing algorithm for wireless sensor Networks with energy efficiency," Automation Instrumentation, vol. 36, no. 12, pp. 4-7, 2015.

6. M. Radhika and P. Sivakumar, "Energy optimized micro genetic algorithm based LEACH protocol for WSN," Wireless Networks, vol. 8, no. 1, 2020.

7. P. Sivakumar and M. Radhika, "Performance analysis of LEACH-GA over LEACH and LEACH-C in WSN," Procedia Computer Science, vol. 125, no. 1, pp. 248-256, 2018.

8. L. Wang, B. J. Xie, Z. Z. Liu et al., "Improved algorithm for non-uniform clustering routing protocol," Computer Scince, vol. 44, no. 2, pp. 152-156, 2017.

9. H. Mostafaei and M. S. Obaidat, "A distributed efficient algorithm for self-protection of wireless sensor networks," in Proceedings of the 2018 IEEE International Conference on Communications (ICC), IEEE, Kansas City, MO, USA, May 2018.

10. L. Deng-Ao, H. Hai-Long, G. Jin-Long et al., "Clustering and Inter-cluster Routing algorithm for wireless sensor Networks with energy efficiency," Automation Instrumentation, vol. 36, no. 12, pp. 4-7, 2015.

11. M. Radhika and P. Sivakumar, "Energy optimized micro genetic algorithm based LEACH protocol for WSN," Wireless Networks, vol. 8, no. 1, 2020.

12. P. Sivakumar and M. Radhika, "Performance analysis of LEACH-GA over LEACH and LEACH-C in WSN," Procedia Computer Science, vol. 125, no. 1, pp. 248-256, 2018.

13. L. Wang, B. J. Xie, Z. Z. Liu et al., "Improved algorithm for non-uniform clustering routing protocol," Computer Scince, vol. 44, no. 2, pp. 152-156, 2017.

14. H. Mostafaei and M. S. Obaidat, "A distributed efficient algorithm for self-protection of wireless sensor networks," in Proceedings of the 2018 IEEE International Conference on Communications (ICC), IEEE, Kansas City, MO, USA, May 2018.

15. K. A. Darabkh, W. S. Al-Rawashdeh, M. Hawa, and S. Ramzi, "M. T.-CHR: A modified threshold-based cluster head replacement protocol for wireless sensor networks," Computers \& Electrical Engineering, vol. 72, pp. 926-938, 2018.

16. Kaur and A. Grover, "LEACH and extended LEACH protocols in wireless sensor network-A survey," International Journal of Computer Applications, vol. 116, no. 10, pp. 1-5, 2015.

17. P. Sivakumar and M. Radhika, "Performance analysis of LEACH-GA over LEACH and LEACH-C in WSN," Procedia Computer Science, vol. 125, 2018. 